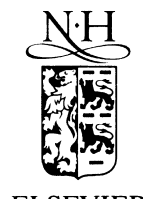

Nuclear Instruments and Methods in Physics Research B 155 (1999) 409-415

\title{
Model for large cluster emission in ion sputtering of metals applied to atomic and polyatomic ion bombardments
}

\author{
S.F. Belykh ${ }^{\text {a }}$, V.I. Matveev ${ }^{\text {b,* }}$, I.V. Veryovkin ${ }^{\text {a }}$, A. Adriaens ${ }^{\text {c }}$, F. Adams ${ }^{\text {c }}$ \\ ${ }^{a}$ Arifov Institute of Electronics of Academy of Sciences, 700143 Tashkent, Uzbekistan \\ b Department of Thermal Physics of Academy of Sciences, Katartal 28, 700135 Tashkent, Uzbekistan \\ c Department of Chemistry of University of Antwerp (UIA), B-2610 Antwerp (Wilrijk), Belgium
}

Received 19 November 1998; received in revised form 13 April 1999

\begin{abstract}
We propose a model for the emission of large clusters $(N \geqslant 5)$ under ion bombardment of metals. In the frame of this model, the relative cluster yields have been calculated as a function of the number of atoms in the cluster for the sputtering of $\mathrm{Al}, \mathrm{Ag}$, Ta and $\mathrm{Nb}$ targets by atomic and polyatomic ions with energies of several keV/atom. The calculated data have shown a very good agreement with experimental results obtained by us and other researchers. On the basis of this agreement we have concluded that larger clusters are emitted as whole agglomerates during the early stages of the collision cascade evolution. () 1999 Elsevier Science B.V. All rights reserved.
\end{abstract}

PACS: $79.20 ; 36.40$

Keywords: Model; Cluster; Emission; Ion; Sputtering; Metals

\section{Introduction}

Fundamental and technological aspects of solid sputtering by energetical ions are widely discussed in the scientific literature (see recent reviews [1-4]). It is well known that the sputtering products consist of a considerable number of the single neutral target atoms (see also experiments $[5,6])$. By using a collision cascade conception, a theory of the sputtering has been developed [7] very well for the

\footnotetext{
${ }^{*}$ Corresponding author. Fax: +7-3712-76-26-68; e-mail: matveev@vict.silk.org
}

emission of single atoms in ion sputtering of solids [1]. However, the sputtering products consist not only of atomic ions and neutrals but represent also a set of polyatomic sputtered particles. The general mechanism for sputtering of diatomic or larger agglomerates of bounded metal atoms (clusters) still remains the subject for discussion [1-4] because there is no satisfactory description of the cluster formation with the number of constituent atoms higher than $5(N \geqslant 5)$. The existing conceptions and models of the cluster formation in sputtering are based on the assumption that this process occurs during late stages of evolution of high density collision cascades in solids. For instance, the 
mixing (overlapping) of several collision cascades, generated by a primary ion and fast recoils, can form either so-called thermal peaks [1], i.e. areas with the high density of energy, or cause a shock wave directed and focused toward the surface [1]. In principle, direct emission of clusters seems to be possible also when target particles obtain energy in the direct collision with the projectile and this leads to a correlated ejection of several particles. During the last decade, "first principles" calculations of cluster emission have become rather popular (see, for instance, [8]) owing to the development of modern computational techniques and effective algorithms for molecular dynamics and MonteCarlo simulations. Unfortunately, such simulations are always very sensitive to potentials describing interatomic and ion-atomic interactions and require exact knowledge of the structure of sputtered solids [1]. Moreover, these simulations become significantly more complicated with the increase in the number of $N$ atoms in sputtered cluster. In such cases, it is necessary to introduce simplifications or approximations into models and they can lead to insufficiently correct results.

In the present work, we propose a model of large cluster emission $(N \geqslant 5)$ under ion bombardment of metals. Being based on simple physical assumptions, this model shows a very good agreement with experiments. On the basis of this agreement we have concluded that larger clusters are emitted as whole agglomerates during the early stages of the collision cascade evolution.

\section{Model}

Let us consider a metal (solids) as a system of harmonic oscillators characterized by the (angular) frequency $\omega$ and localized within potential wells with the depth $\Delta$. This corresponds to the cut-off harmonic oscillator potential with the oscillation period $T=2 \pi / \omega .^{1}$

\footnotetext{
${ }^{1}$ Note that we consider all states to be equidistant. It is possible when the total number of considered bound states is sufficiently large (in our case we have order of $\sim 10^{2}-10^{3}$ states) so that we can neglect the possible non-equidistantness in the neighbourhood of the cut-off boundaries.
}

For primary ions (or for any fast recoils), we suppose their velocity to be sufficient to undergo many collisions with atoms of the metal for the time of $\tau \ll T$. In this case the metal atoms obtain some momentums $\mathbf{q}_{i}$, where $i$ is the number of atom. That is, the oscillator with the number $i$ obtained the momentum $\mathbf{q}_{i}$, the oscillator with the number $j$ obtained the momentum $\mathbf{q}_{j}$ and, generally speaking, $\mathbf{q}_{i} \neq \mathbf{q}_{j}$, if $i \neq j$. The condition $\tau \ll T$ means that the primary ion penetration through the oscillators system results in the simultaneous and instantaneous transfer of momentums $\mathbf{q}_{i}$ to each oscillator $(i=1,2, \ldots, N$, where $N$ is the number of oscillators).

Let us formulate conditions when such a selected group of $N$ atoms can move as a whole agglomerate or be emitted as a cluster:

1. All atoms have the same average values of the momentum $\mathbf{k} / N$ so that the total momentum of the cluster is equal to $\mathbf{k}$.

2. Kinetic energy of the agglomerate (cluster) motion is sufficient to break the bounding of this cluster with the metal.

3. The energy of the vibrational excitation of the oscillators is insufficient for the destruction (fragmentation) of the cluster.

To calculate the probability of such events of the correlated motion (emission) of the selected group of atoms, it is easier to use the quantum mechanics. ${ }^{2}$ We will express the wave function of the system of $N$ oscillators as a product of $N$ wave functions corresponding to each oscillator. For the random stationary state of a three-dimensional isotropic oscillator with the index $i$ and the average momentum $\mathbf{k} / N$, the following wave function will be used: ${ }^{3}$

\footnotetext{
${ }^{2}$ Note that we use quantum mechanics frames not because of any "higher precision" of calculations but just because the solution of the task can be achieved relatively easier compared to classical mechanics.

${ }^{3}$ To avoid misunderstandings, it should be emphasized that the wave function (1) is not a function for the states of the continuous spectrum of the isolated oscillator (the cut-off oscillator potential). This function corresponds to the fact that, being in the bound state $\left|n_{i x}, n_{i y}, n_{i z}\right\rangle$, this oscillator is moving as a whole with the average momentum $\mathbf{k} / N$ together with other $(N-1)$ oscillators.
} 
$\exp \left(\mathrm{i} \frac{\mathbf{k}}{\hbar N} \mathbf{r}_{i}\right)\left|n_{i x}, n_{i y}, n_{i z}\right\rangle$,

where $\mathbf{r}_{i}$ are coordinates of the oscillator with index $i$, and $n_{i x}, n_{i y}, n_{i z}$ are quantum numbers. The energy of this state can be expressed by using the principal quantum number $n_{i}=n_{i x}+n_{i y}+n_{i z}$ as follows: $\varepsilon_{n i}=\hbar \omega\left(n_{i}+3 / 2\right)+k^{2} /\left(2 m N^{2}\right)$. We assume, before obtaining the momentum $\mathbf{q}_{i}$, each oscillator to be in the ground state $|0,0,0\rangle$. After the sudden obtaining of the momentum $\mathbf{q}_{i}$, the oscillator wave function will be $\exp \left(\mathbf{i q}_{i} \mathbf{r}_{\mathbf{i}}\right)|0,0,0\rangle$. If we have the system of $N$ oscillators each of which the momentum $\mathbf{q}_{i}, i=(1,2, \ldots, N)$ is suddenly transferred to, then the probability to find the system in any of states with the energy $E_{n}=\hbar \omega(n+N 3 / 2)+k^{2} /(2 m N)$, where $n=\sum_{i=1}^{N} n_{i}$, and thus system being moved as a whole agglomerate (cluster) with the total momentum $\mathbf{k}=N(\mathbf{k} / N)$ can be expressed as follows:

$$
\begin{aligned}
W_{N}^{(n)}= & \frac{1}{n !}\left[\frac{1}{2 \alpha^{2} \hbar^{2}} \sum_{i=1}^{N}\left(\mathbf{q}_{i}-\frac{\mathbf{k}}{N}\right)^{2}\right]^{n} \\
& \times \exp \left\{-\frac{1}{2 \alpha^{2} \hbar^{2}} \sum_{i=1}^{N}\left(\mathbf{q}_{i}-\frac{\mathbf{k}}{N}\right)^{2}\right\},
\end{aligned}
$$

where $\alpha^{2}=m \omega / \hbar$. To calculate the matrix element square and the sums, it is possible to use results obtained by Amaldy and Fermi [9] (see also [10]).

Next, one should calculate a sum of all the probabilities (2) over all vibrational excitation states of the cluster up to value of $n=n_{0} \approx \Delta / \hbar \omega$ when the energy accumulated by excited oscillators is sufficient for the cluster fragmentation. For the summing we used (for $n_{0} \gg 1$ ) the approximation $\sum_{n=0}^{n_{0}} \approx \sum_{n=0}^{\infty} \exp \left\{-n / n_{0}\right\}$.

The next step is to average the probability over all possible values of $\mathbf{q}_{i}, i=(1,2, \ldots, N)$. For the distribution of $\mathbf{q}_{i}$ values, we assume all of them to be independent and all directions of $\mathbf{q}_{i}$ vectors equally probable.

It is possible to simplify the further calculations by assuming that all $\mathbf{q}_{i}$ vectors also have the equal length: ${ }^{4}\left|\mathbf{q}_{i}\right|=q$ but $\mathbf{q}_{i} \neq \mathbf{q}_{j}$, if $i \neq j$ (i.e. in average, all $\mathbf{q}_{i}$ vectors have equal values but directed chaotically). The obtained expression will describe the probability of ejection (emission) of $\mathrm{N}$-atomic agglomerate (cluster) if the kinetic energy of the cluster is sufficient to get over the binding energy with other atoms of the metal. The binding energy of $N$-atomic cluster with the metal is proportional to the surface area $S_{N}$ of the contact between the metal and the selected $N$-atomic agglomerate (cluster). Let us consider this area (before sputtering) as a semi-sphere with the centre placed on the metal surface. Then the binding surface energy of the cluster is

$$
\begin{aligned}
U_{N}=\sigma S_{N}=\sigma 2 \pi R_{N}^{2} & =\sigma 2 \pi\left(\frac{3}{2 \pi d}\right)^{2 / 3} N^{2 / 3} \\
& =\delta N^{2 / 3}
\end{aligned}
$$

where $R_{N}$ is the radius of the semi-sphere, $d$ the number of atoms in the volume unit and $\delta$ is, obviously, the binding cluster-metal energy related to one atom of the cluster.

If, before being emitted from the surface, the cluster obtains the momentum $\mathbf{k}$, then after this emission event the cluster will be moving with the kinetic energy $T_{N}=k^{2} /(2 m N)-U_{N}$. Assuming $T_{N}=0$, we can obtain the minimal value of the momentum $k_{0 N}$ necessary for getting over the binding surface energy: $k_{0 N}=\left[2 m N \sigma S_{N}\right]^{1 / 2}=$ $[2 m \delta]^{1 / 2} N^{5 / 6}=k_{01} N^{5 / 6}$, where $k_{01}=(2 m \delta)^{1 / 2}$ is the minimal momentum per atom of the cluster which is necessary for its separation (emission) from the surface. To calculate the probability of the $N$-atomic cluster emission, we should integrate over all possible allowed values of $\mathbf{k}$ vector: i.e. when $\mathbf{k}$ has lengths of $|\mathbf{k}|\rangle k_{0 N}$ and it is directed outside the metal (this corresponds to the solid angle of $2 \pi$ ). As a result, we obtain the final expression for the probability of the emission of the $N$-atomic cluster:

\footnotetext{
${ }^{4}$ This very statement cancels the upper limitation to the number of atoms in the cluster. Nevertheless, within the range of $N$ which we used to make a comparison between the theory and experiments, this condition has been satisfied.
} 


$$
\begin{aligned}
W_{N}= & 2 \pi \int_{0}^{\infty} k m N \mathrm{~d} T_{N}\left[4 \frac{q}{k_{01}} \frac{k}{N k_{01}} \frac{\delta}{\Delta}\right]^{-N} \\
& \times\left[\exp \left\{-\frac{\delta}{\Delta}\left(\frac{q}{k_{01}}-\frac{k}{N k_{01}}\right)^{2}\right\}\right. \\
& \left.-\exp \left\{-\frac{\delta}{\Delta}\left(\frac{q}{k_{01}}+\frac{k}{N k_{01}}\right)^{2}\right\}\right]^{N},
\end{aligned}
$$

where $\quad k=\sqrt{2 m N T_{N}+\left(k_{0 N}\right)^{2}}, k_{0 N}=k_{01} N^{v}, v=$ $5 / 6$, taking into account that $\beta k_{01}^{2} / n_{0}=\delta / \Delta$, and $q \geqslant k_{01} N^{v-1}$.

Thus, we have calculated the probability of the emission of the cluster as a whole agglomerate of atoms without changing of mutual positions of the atoms. The case of changing atomic positions would be analogous to the cluster formation from separately sputtered atoms. If we take into account also such a possibility, then it would lead to the appearance of an additional small parameter corresponding to overlapping of oscillator wave functions which are centred in different places. Therefore, we can neglect such a case. It is obvious that our model is not applicable for the sputtering of single atoms or small clusters, in principle.

\section{Results and discussion}

It should be noted that integration procedures performed over variables $\mathbf{k}$ transform the non-dimensional probability (which is lower than unity) into non-normalized dimensional probability. But it is insignificant for the comparison with experiments in which the relative yield intensities for clusters having the different numbers of atoms are measured.

Moreover, it should be emphasized that we consider emission processes for large clusters with $N \gg 1$, and from comparison with experiment we obtained that $N \geqslant 5$. For us, it was appropriate to select the cluster with $N=5$, for such a normalizing procedure the relative yield is $Y_{N}=W_{N} / W_{5}$. Experimental data should be normalized by the same way. After such a normalization, any other appropriate (arbitrary) units can be used. We calculated normalized yields $Y_{N}$ of clusters for a number of elements sputtered by primary $\mathrm{Ar}^{+}$ions of $5 \mathrm{keV}$. In order to use the minimally possible number of adjustment (variable) parameters in our model, we set $\Delta=\delta$ and only $q$ values were varied. All calculations have been performed for atomic units system: $\hbar=m_{e}=e=1$.

We show the calculation procedure for formula (4) for the Ta-target sputtering by the primary $\mathrm{Ar}^{+}$ ions of $5 \mathrm{keV}$ : in atomic units, the Ta atom mass is $\approx 181 \times 1.8 \times 10^{3} ; \delta$ is the sublimation energy equal to $8.675 \mathrm{eV} \approx 8.675 / 27.2$ a.u. [11]; $k_{01}=$ $\sqrt{2 m \delta}, k_{0 N}=k_{01} N^{5 / 6}$ and $k=\sqrt{2 m N T_{N}+\left(k_{0 N}\right)^{2}}$. Now for the given values $N \geqslant 5$ and $q$ we can integrate formula (4) over $\mathrm{d} T_{N}$ and find $W_{N}$ and $Y_{N}=W_{N} / W_{5}$ (for example, for $N=10, q=550$ and $\Delta=\delta$ we obtained $Y_{10}=W_{10} / W_{5} \approx 0.003$ ).

Experimental data for sputtered cluster neutrals and the corresponding model calculation results

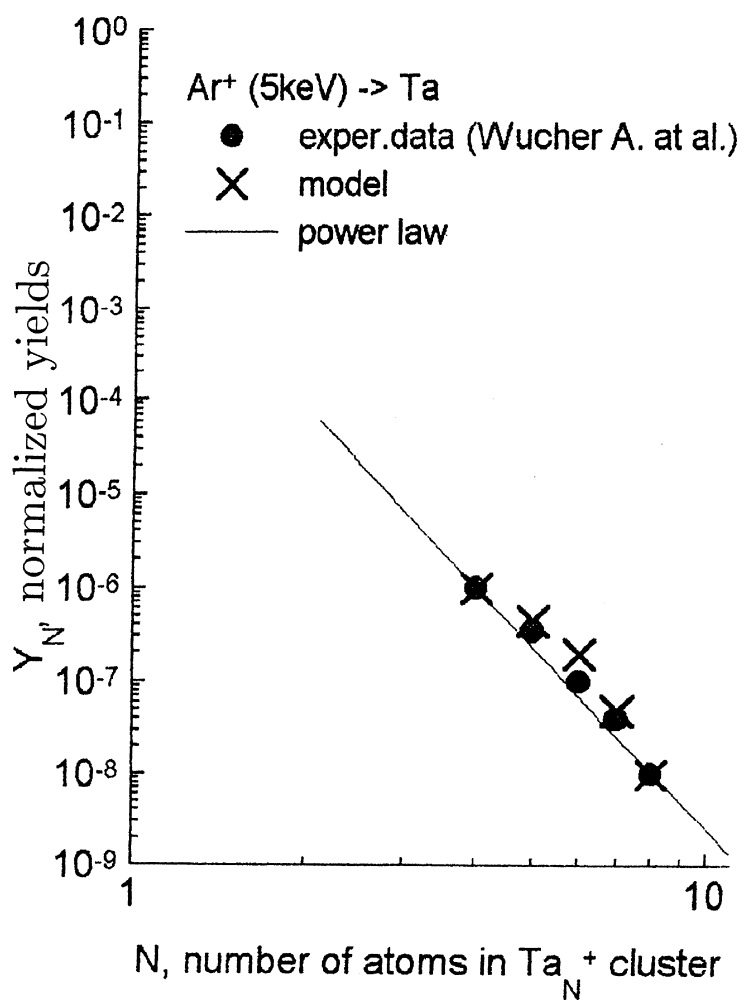

Fig. 1. The normalized yields $Y_{N}$ of clusters as function of the number $N$ of atoms in the cluster, for the sputtering of Ta target by the $5 \mathrm{keV}$ primary $\mathrm{Ar}^{+}$ions. $(\times)$theory, $(\bullet)$ experiment [5], solid line - power law [5]. 


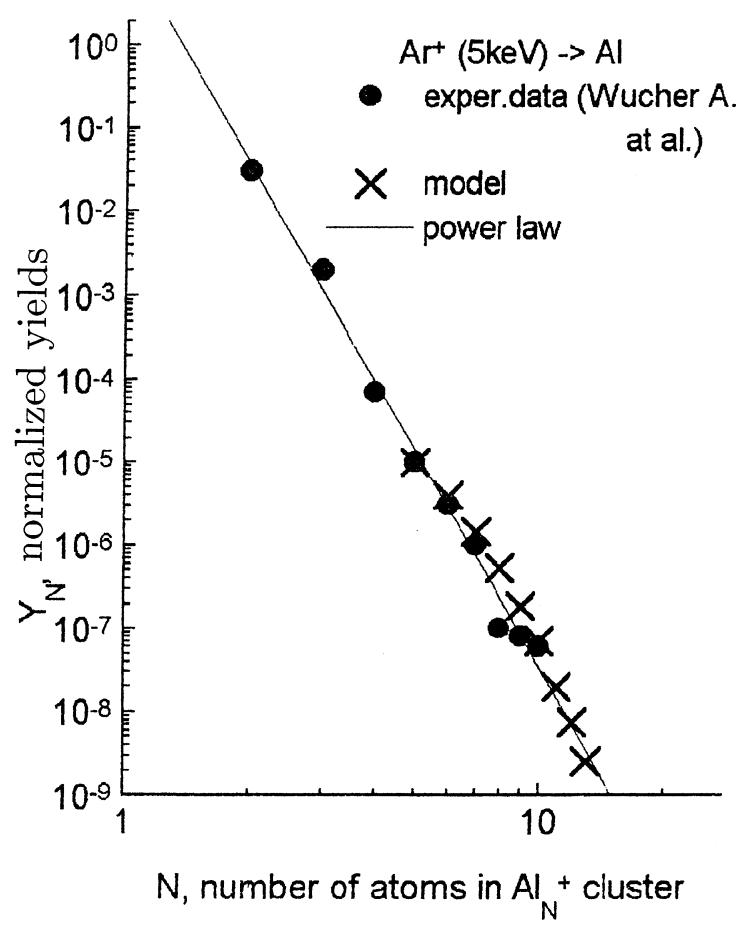

Fig. 2. The normalized yields $Y_{N}$ of clusters as function of the number $N$ of atoms in the cluster, for the sputtering of $\mathrm{Al}$ target by the $5 \mathrm{keV}$ primary $\mathrm{Ar}^{+}$ions. $(\times)$theory, $(\bullet)$ experiment [5], solid line - power law [5].

are shown in Figs. 1-4. It should be noted that a good agreement has been observed at the values of $q$ being close to $k_{01}=\sqrt{2 m \delta}$.

The independence of the ionization degree of large clusters on the number of atoms in the cluster has been shown in Ref. [12] for $N \geqslant 5$. Therefore, relative cluster yields do not depend on the ionization degree and should have the same values for cluster neutrals and cluster ions. Supposing this, we have applied our model for the sputtering of cluster ions. Fig. 5 shows the comparison between our experimental results on $\mathrm{Ta}_{N}^{+}$clusters sputtered by $(6 \mathrm{keV} /$ atom $)$ atomic and polyatomic $\mathrm{Au}_{m}^{-}$ions $(m=1,2,3)[13,14]$ and the corresponding calculation results obtained by the present model. Additionally, results on cluster $\mathrm{Ta}_{N}$ neutrals sputtered by $\mathrm{Ar}^{+}$ions [5] are shown in Fig. 5 .

Our model made a distinction between parameters $\Delta$ (the depth of the potential well where each atom of solid is localized) and $\delta$ (the binding

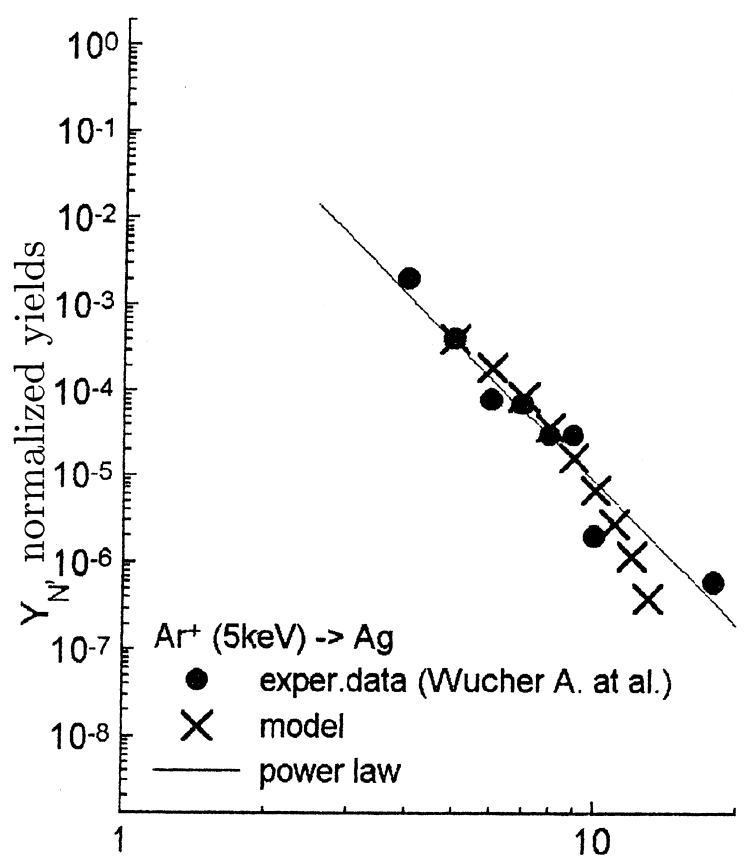

$\mathrm{N}$, number of atoms in $\mathrm{Ag}_{\mathrm{N}}{ }^{+}$cluster

Fig. 3. The normalized yields $Y_{N}$ of clusters as function of the number $N$ of atoms in the cluster, for the sputtering of $\mathrm{Ag}$ target by the $5 \mathrm{keV}$ primary $\mathrm{Ar}^{+}$ions. $(\times)$theory, $(\bullet)$ experiment [5], solid line - power law [5].

cluster-metal energy per one atom of the cluster). As a traditional approximation, we used tabulated literature data for the sublimation energies to estimate the values of the binding surface energy $\delta$. To clarify the role of the parameter $\delta$, we performed calculations of the probability (4) when the $\delta$ variable is decreasing at fixed value of the $\Delta$ parameter: $\Delta_{\mathrm{Ta}}=\delta_{\mathrm{Ta}}=8.675 \mathrm{eV}$. For the bombardment by polyatomic primary ions, we have observed the best agreement between the present model and experiment [13,14] when $\delta$-parameter was reduced as follows: for primary $\mathrm{Au}_{2}^{-}$ions $-\delta=0.73 \delta_{\mathrm{Ta}}=6.33 \mathrm{eV}$ and for primary $\mathrm{Au}_{3}^{-}$ions $-\delta=0.56 \delta_{\mathrm{Ta}}=4.86 \mathrm{eV}$. We made the same calculations for sputtering of $\mathrm{Nb}$. The corresponding theoretical calculations and experimental data [15] are shown in Fig 6. Additionally, results on cluster $\mathrm{Nb}_{N}$ neutrals sputtered by $\mathrm{Ar}^{+}$ions [5] are also shown in Fig. 6. In Table 1 


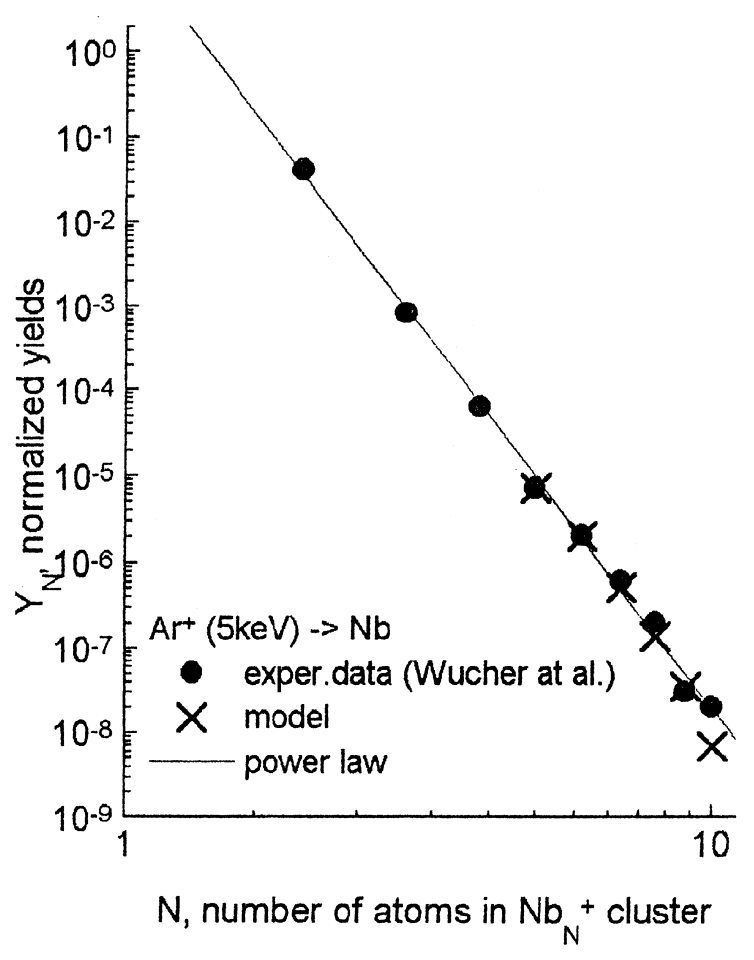

Fig. 4. The normalized yields $Y_{N}$ of clusters as function of the number $N$ of atoms in the cluster, for the sputtering of $\mathrm{Nb}$ targets by the $5 \mathrm{keV}$ primary $\mathrm{Ar}^{+}$ions. $(\times)$theory, $(\bullet)$ experiment [5], solid line - power law [5].

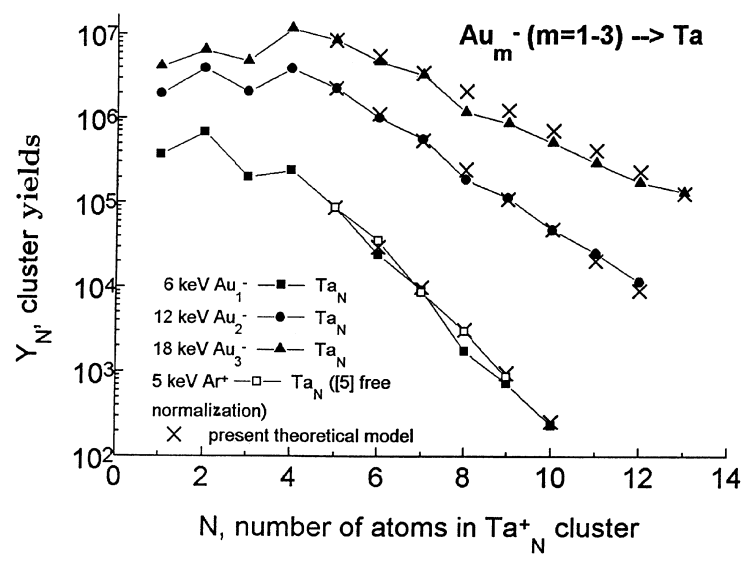

Fig. 5. The cluster yields $Y_{N}$ as function of the number $N$ of atoms in the cluster, for the sputtering of Ta target by atomic and cluster ions with the energy $6 \mathrm{keV}$ per atom: $(\boldsymbol{\Lambda}) \mathrm{Au}_{3}^{-} ;(\bullet)$

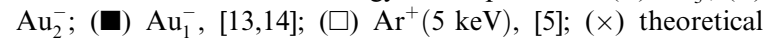
results.

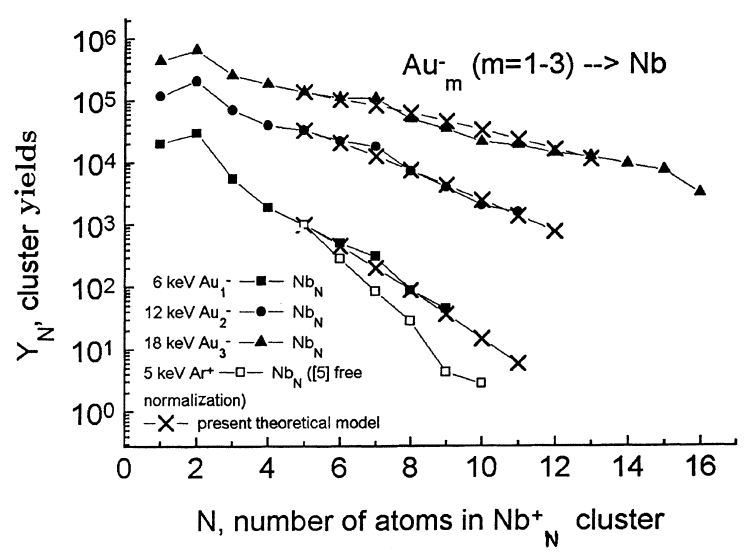

Fig. 6. The cluster yields $Y_{N}$ as function of the number $N$ of atoms in the cluster, for the sputtering of $\mathrm{Nb}$ target by atomic and cluster ions with the energy $6 \mathrm{keV}$ per atom: $(\boldsymbol{\Lambda}) \mathrm{Au}_{3}^{-} ;(\bullet)$ $\mathrm{Au}_{2}^{-}$; (匚) $\mathrm{Au}_{1}^{-},[15] ;(\square) \mathrm{Ar}^{+}(5 \mathrm{keV}),[5] ;(\times)$ theoretical results.

Table 1

\begin{tabular}{|c|c|c|c|}
\hline Primary ion & Target & $\delta(\mathrm{eV})$ & $q$ (at. un.) \\
\hline $\mathrm{Ar}^{+}(5 \mathrm{keV})$ & $\mathrm{Ag}$ & 2.96 from [19] & 200 \\
\hline $\mathrm{Ar}^{+}(5 \mathrm{keV})$ & $\mathrm{Al}$ & 3.34 from [19] & 120 \\
\hline $\mathrm{Ar}^{+}(5 \mathrm{keV})$ & $\mathrm{Ta}$ & $\begin{array}{l}8.675 \text { from } \\
{[11]}\end{array}$ & 550 \\
\hline $\mathrm{Au}_{1}^{-}(6 \mathrm{keV})$ & $\mathrm{Ta}$ & $\begin{array}{l}8.675 \text { from } \\
{[11]}\end{array}$ & 550 \\
\hline $\mathrm{Au}_{2}^{-}(6 \mathrm{keV} /$ atom $)$ & $\mathrm{Ta}$ & $6.33\left(0.73 \delta_{\mathrm{Ta}}\right)$ & 450 \\
\hline $\mathrm{Au}_{3}^{-}(6 \mathrm{keV} /$ atom $)$ & $\mathrm{Ta}$ & $4.86\left(0.56 \delta_{\mathrm{Ta}}\right)$ & 370 \\
\hline $\mathrm{Ar}^{+}(5 \mathrm{keV})$ & $\mathrm{Nb}$ & 7.47 from [19] & 400 \\
\hline $\mathrm{Au}_{1}^{-}(6 \mathrm{keV})$ & $\mathrm{Nb}$ & 7.47 from [19] & 300 \\
\hline $\mathrm{Au}_{2}^{-}(6 \mathrm{keV} /$ atom $)$ & $\mathrm{Nb}$ & $5.45\left(0.73 \delta_{\mathrm{Nb}}\right)$ & 240 \\
\hline $\mathrm{Au}_{3}^{-}(6 \mathrm{keV} /$ atom $)$ & $\mathrm{Nb}$ & $4.2\left(0.56 \delta_{\mathrm{Nb}}\right)$ & 190 \\
\hline
\end{tabular}

the values of the parameter $\delta$ and the variable parameter $q$ for our calculations are presented.

In our opinion, this indicates that the dramatic increase of the large cluster emission under polyatomic ion bombardment observed in Refs. [13$15]$ is the consequence of lowering the binding cluster-metal energy affected by the polyatomic projectiles. Earlier such a hypothesis about a lowering of the binding energy was proposed by Johar and Thompson [16] to explain the fact that the total yield of sputtering from metals $(\mathrm{Ag}, \mathrm{Au}$ and $\mathrm{Pt}$ ) under polyatomic $\mathrm{P}_{2}^{+}, \mathrm{As}_{2}^{+}, \mathrm{Sb}_{2}^{+}$and $\mathrm{Bi}_{2}^{+}$ ion bombardment exceeds (approximately by several times) the yield under atomic $\mathrm{P}_{1}^{+}, \mathrm{As}_{1}^{+}, \mathrm{Sb}_{1}^{+}$ 
and $\mathrm{Bi}_{1}^{+}$ion bombardment over the energy range $10-250 \mathrm{keV}$. However, the sputtering of large clusters, rather than the sputtering of single atoms, is more sensitive to the number of atoms in the projectile [13-15,17,18]. For example, the emission enhancement for $\mathrm{Ta}_{10}^{+}$clusters (when triatomic $\mathrm{Au}_{3}^{-}$projectiles are compared with atomic $\mathrm{Au}_{1}^{-}$ ones) was observed to be three orders of a magnitude [13]. It should be noted that this emission enhancement for large clusters under the polyatomic ion bombardment was observed under different experimental conditions (see, also $[17,18]$ ) and this is seemingly a general trend of polyatomic ion bombardment.

Thus, the comparison of results of our model with those observed in experiments on sputtering of cluster ions and cluster neutrals shows a good agreement within the range of large clusters with $(N \geqslant 5)$. This allows us to make the following conclusion: mechanisms responsible for the emission of small clusters $(N<5)$ and larger ones $(N \geqslant 5)$ are significantly different. For small clusters, direct emission and any (re)combination mechanisms, traditionally discussed in the literature, are possible for the whole time of the collision cascade evolution (usually this is the time $\geqslant 10^{-12} \mathrm{~s}$ ). In contrast, larger clusters are emitted as whole agglomerates during the early stages of the collision cascade for times $\leqslant 10^{-13} \mathrm{~s}$.

\section{Acknowledgements}

This study was partially supported by the following organizations: Fundamental Research Support Foundation of the Academy of Sciences
(Grant 29-96, Uzbekistan) and INTAS (Project 96-0470).

\section{References}

[1] H.M. Urbassek, W.O. Hofer, K. Dan, Vidensk. Selsk. Mat. Fys. Medd. 43 (1993) 97.

[2] H.H. Andersen, K. Dan, Vidensk. Selsk. Mat. Fys. Medd. 43 (1993) 127.

[3] I.A. Baranov, U.U. Martinenko, S.O. Tcepelevich, U.N. Yavlinsky, Usp. Fiz. Nauk 156 (1998) 478.

[4] G. Betz, W. Wahl, Int. J. Spectr. Ion Proc. 140 (1994) 1.

[5] A. Wucher, W. Wahl, Nucl. Instr. and Meth. B 115 (1996) 581.

[6] S.R. Coon, W.F. Calaway, M.Y. Pellin, Nucl. Instr. and Meth. B 90 (1994) 518.

[7] P. Sigmund, Phys. Rev. 184 (1969) 383.

[8] A. Wucher, B.Y. Garrison, J. Chem. Phys. 105 (1996) 5999.

[9] E. Amaldi, E. Fermi, Phys. Rev. 50 (1936) 899.

[10] L.D. Landau, E.M. Lifshitz, Quantum Mechanics, Pergamon Press, Oxford, 1977 [Russ. oridginal, 4th ed., Nauka, Moskow, 1989].

[11] E. Langberg, Phys. Rev. 111 (1956) 91.

[12] W. Wahl, A. Wucher, Nucl. Instr. and Meth. B 94 (1994) 36.

[13] S.F. Belykh, U.Kh. Rasulev, A.V. Samartsev, I.V. Veryovkin, Nucl. Instr. and Meth. B 136-138 (1998) 773.

[14] S.F. Belykh, U.Kh. Rasulev, A.V. Samartsev, S.V. Verkhoturov, I.V. Veryovkin, Mikrochim. Acta 15 (Suppl.) (1998) 379.

[15] S.F. Belykh, B. Habets, U.Kh. Rasulev, A.V. Samartsev, L.V. Stroev, I.V. Veryovkin, Abstract Book of SIMS Europe 1998, Muenster, Germany, 4-6 October 1998, p. 5.

[16] S.S. Johar, D.A. Thompson, Surf. Sci. 90 (1979) 319.

[17] A. Brunelle, S. Della-Negra, C. Deprun, J. Depauw, P. Hakansson, D. Jacquet, Y. Le Beyec, Int. J. Mass. Spectr. Ion Proc. 164 (1997) 193.

[18] Y. Le Beyec, Int. J. Mass. Spectr. Ion Proc. 174 (1998) 101.

[19] C. Kittel, Introduction to Solid State Physics, 4th ed., Wiley, New York, 1963. 